\title{
Endovascular Treatment of Acute Ischemic Stroke: Ready to Go?
}

\author{
M. Bendszus $\cdot$ S. Rohde
}

Published online: 2 April 2011

(C) Springer-Verlag 2011

Even though intra-arterial fibrinolysis has shown a significantly better outcome in the Prolyse in Acute Cerebral Thromboembolism II (PROACT II) trial, this treatment option was not approved by the US Food and Drug Administration (FDA), whereas intravenous fibrinolysis was approved as a standard therapy. New mechanical endovascular approaches, such as the Merci device or the Penumbra system were initially seen with great enthusiasm in the neuro-interventional community as high recanalization rates of up to $80 \%$ were achieved. Nevertheless, clinical results were disappointing which is why these techniques were increasingly critically viewed by stroke neurologists. In recent years a new technique with stent-like retrievers, has changed the practice in many stroke centers. In single center experiences even higher recanalization rates have been reported which were also associated with improved outcome in a substantial number of patients. This is a positive development which also has to be addressed with great care, though, as clinical neuroradiologists have to show improved outcomes in a similar fashion to stroke neurologists, i.e. in randomized clinical trials otherwise the goal of a broad and established interventional stroke treatment will never be achieved. This goal, however, comes with another challenge: at present, interventional neuroradiology is focused on a few large centers and there are far too few neuroradiologists able to perform interventional procedures, such as mechanical thrombectomy. If there is belief in this treatment it also has to be believed that it will become a standard treatment in cases of acute large cerebral vessel occlusion. In this case there will be a large need for trained physicians to perform these procedures all over the country. If interventional neuroradiology does not manage to train enough neuroradiologists who are able to perform these procedures other specialities will discover this treatment option for themselves.
M. Bendszus $(\bowtie) \cdot$ S. Rohde

Dept. of Neuroradiology, Heidelberg, Deutschland

e-mail: martin_bendszus@med.uni-heidelberg.de 